\title{
Versatile supercontinuum generation in parabolic multimode optical fibers
}

\author{
M. A. EfTEKhAR, ${ }^{1,{ }^{*}}$ L. G. Wright, ${ }^{2}$ M. S. Mills, ${ }^{1}$ M. KOLESIK, ${ }^{3}$ R. \\ Amezcua CorReA, ${ }^{1}$ F. W. Wise, ${ }^{2}$ AND D. N. Christodoulides ${ }^{1}$ \\ ${ }^{1}$ CREOL, College of Optics and Photonics, University of Central Florida, Orlando, Florida 32816- \\ 2700, USA \\ ${ }^{2}$ School of Applied and Engineering Physics, Cornell University, Ithaca, New York 14853, USA \\ ${ }^{3}$ The College of Optical Sciences, The University of Arizona, Tucson, AZ 85721, USA \\ *m.a.eftekhar@knights.ucf.edu
}

\begin{abstract}
We demonstrate that the pump's spatial input profile can provide additional degrees of freedom in tailoring at will the nonlinear dynamics and the ensuing spectral content of supercontinuum generation in highly multimoded optical fibers. Experiments and simulations carried out at $1550 \mathrm{~nm}$ indicate that the modal composition of the input beam can substantially alter the soliton fission process as well as the resulting Raman and dispersive wave generation that eventually lead to supercontinuum in such a multimode environment. Given the multitude of conceivable initial conditions, our results suggest that it is possible to pre-engineer the supercontinuum spectral content in a versatile manner.
\end{abstract}

(C) 2017 Optical Society of America

OCIS codes: (060.4370) Nonlinear optics, fibers; (190.4380) Nonlinear optics, four-wave mixing; (320.6629) Supercontinuum generation.

\section{References and links}

1. R. R. Alfano, The Supercontinuum Laser Source: The Ultimate White Light, 3rd ed. (Springer, 2016).

2. J. K. Ranka, R. S. Windeler, and A. J. Stentz, "Visible continuum generation in air-silica microstructure optical fibers with anomalous dispersion at $800 \mathrm{~nm}$," Opt. Lett. 25(1), 25-27 (2000).

3. W. Wadsworth, N. Joly, J. Knight, T. Birks, F. Biancalana, and P. Russell, "Supercontinuum and four-wave mixing with Q-switched pulses in endlessly single-mode photonic crystal fibres," Opt. Express 12(2), 299-309 (2004).

4. J. M. Dudley, G. Genty, and S. Coen, "Supercontinuum generation in photonic crystal fiber," Rev. Mod. Phys. 78(4), 1135-1184 (2006).

5. J. M. Stone and J. C. Knight, "Visibly "white" light generation in uniform photonic crystal fiber using a microchip laser," Opt. Express 16(4), 2670-2675 (2008).

6. C. Sun, T. Ge, S. Li, N. An, K. Cao, and Z. Wang, "53.3 W visible-waveband extra high power supercontinuum all-fiber laser," IEEE Photonics J. 8, 1-7 (2016).

7. D. J. Richardson, J. M. Fini, and L. E. Nelson, "Space-division multiplexing in optical fibres," Nat. Photonics 7(5), 354-362 (2013)

8. J. van Weerdenburg, A. Velàzquez-Benitez, R. van Uden, P. Sillard, D. Molin, A. Amezcua-Correa, E. AntonioLopez, M. Kuschnerov, F. Huijskens, H. de Waardt, T. Koonen, R. Amezcua-Correa, and C. Okonkwo, "10 Spatial mode transmission using low differential mode delay 6-LP fiber using all-fiber photonic lanterns," Opt. Express 23(19), 24759-24769 (2015).

9. P. Sillard, D. Molin, M. Bigot-Astruc, K. D. Jongh, F. Achten, A. M. Velázquez-Benítez, R. Amezcua-Correa, and C. M. Okonkwo, "Low-differential-mode-group-delay 9-LP-mode fiber," J. Lightwave Technol. 34(2), 425430 (2016).

10. R. H. Stolen, J. E. Bjorkholm, and A. Ashkin, "Phase-matched three-wave mixing in silica fiber optical waveguides," Appl. Phys. Lett. 24(7), 308-310 (1974).

11. R. Stolen, "Phase-matched-stimulated four-photon mixing in silica-fiber waveguides," IEEE J. Quantum Electron. 11(3), 100-103 (1975).

12. R. Stolen and J. Bjorkholm, "Parametric amplification and frequency conversion in optical fibers," IEEE J. Quantum Electron. 18(7), 1062-1072 (1982).

13. A. Hasegawa, "Self-confinement of multimode optical pulse in a glass fiber," Opt. Lett. 5(10), 416-417 (1980).

14. B. Crosignani and P. Di Porto, "Soliton propagation in multimode optical fibers," Opt. Lett. 6(7), 329-330 (1981).

15. A. B. Grudinin, E. M. Dianov, D. V. Korbkin, A. M. Prokhorov, and D. V. Khaidarov, "Nonlinear mode coupling in multimode optical fibers; excitation of femtosecond-range stimulated-Raman-scattering solitons," Sov. J. Exp. Theor. Phys. Lett. 47, 356 (1988). 
16. K. S. Chiang, "Stimulated Raman scattering in a multimode optical fiber: evolution of modes in Stokes waves," Opt. Lett. 17(5), 352-354 (1992).

17. N. B. Terry, T. G. Alley, and T. H. Russell, "An explanation of SRS beam cleanup in graded-index fibers and the absence of SRS beam cleanup in step-index fibers," Opt. Express 15(26), 17509-17519 (2007).

18. S. Mumtaz, R.-J. Essiambre, and G. P. Agrawal, "Nonlinear propagation in multimode and multicore fibers: generalization of the Manakov equations," J. Lightwave Technol. 31(3), 398-406 (2013).

19. H. Pourbeyram, G. P. Agrawal, and A. Mafi, "Stimulated Raman scattering cascade spanning the wavelength range of 523 to $1750 \mathrm{~nm}$ using a graded-index multimode optical fiber," Appl. Phys. Lett. 102(20), 201107 (2013).

20. J. Demas, P. Steinvurzel, B. Tai, L. Rishøj, Y. Chen, and S. Ramachandran, "Intermodal nonlinear mixing with Bessel beams in optical fiber," Optica 2(1), 14 (2015).

21. F. Poletti and P. Horak, "Description of ultrashort pulse propagation in multimode optical fibers," J. Opt. Soc. Am. B 25(10), 1645 (2008).

22. A. Picozzi, G. Millot, and S. Wabnitz, "Nonlinear optics: Nonlinear virtues of multimode fibre," Nat. Photonics 9(5), 289-291 (2015)

23. W. H. Renninger and F. W. Wise, "Optical solitons in graded-index multimode fibres," Nat. Commun. 4, 1719 (2013).

24. L. G. Wright, W. H. Renninger, D. N. Christodoulides, and F. W. Wise, "Spatiotemporal dynamics of multimode optical solitons," Opt. Express 23(3), 3492-3506 (2015).

25. B. Crosignani, A. Cutolo, and P. Di Porto, "Coupled-mode theory of nonlinear propagation in multimode and single-mode fibers: envelope solitons and self-confinement," J. Opt. Soc. Am. 72(9), 1136-1141 (1982).

26. L. G. Wright, D. N. Christodoulides, and F. W. Wise, "Controllable spatiotemporal nonlinear effects in multimode fibres," Nat. Photonics 9(5), 306-310 (2015).

27. L. G. Wright, S. Wabnitz, D. N. Christodoulides, and F. W. Wise, "Ultrabroadband dispersive radiation by spatiotemporal oscillation of multimode waves," Phys. Rev. Lett. 115(22), 223902 (2015).

28. G. Lopez-Galmiche, Z. Sanjabi Eznaveh, M. A. Eftekhar, J. Antonio Lopez, L. G. Wright, F. Wise, D. Christodoulides, and R. Amezcua Correa, "Visible supercontinuum generation in a graded index multimode fiber pumped at $1064 \mathrm{~nm}, "$ Opt. Lett. 41(11), 2553-2556 (2016).

29. S. Longhi, "Modulational instability and space-time dynamics in nonlinear parabolic-index optical fibers," Opt. Lett. 28(23), 2363-2365 (2003).

30. K. Krupa, A. Tonello, A. Barthélémy, V. Couderc, B. M. Shalaby, A. Bendahmane, G. Millot, and S. Wabnitz, "Observation of geometric parametric instability induced by the periodic spatial self-Imaging of multimode waves," Phys. Rev. Lett. 116(18), 183901 (2016).

31. K. Krupa, A. Tonello, B. M. Shalaby, M. Fabert, A. Barthélémy, G. Millot, S. Wabnitz, and V. Couderc, "Spatial beam self-cleaning in multimode fiber," ArXiv160302972 Phys. (2016).

32. J. Andreasen and M. Kolesik, "Nonlinear propagation of light in structured media: Generalized unidirectional pulse propagation equations," Phys. Rev. E Stat. Nonlin. Soft Matter Phys. 86(3), 036706 (2012).

33. M. Kolesik and J. V. Moloney, "Nonlinear optical pulse propagation simulation: From Maxwell's to unidirectional equations," Phys. Rev. E Stat. Nonlin. Soft Matter Phys. 70(3 Pt 2), 036604 (2004).

34. D. Hollenbeck and C. D. Cantrell, "Multiple-vibrational-mode model for fiber-optic Raman gain spectrum and response function,” J. Opt. Soc. Am. B 19(12), 2886-2892 (2002).

35. A. W. Snyder and J. Love, Optical Waveguide Theory (Springer Science \& Business Media, 2012).

36. J. Towns, T. Cockerill, M. Dahan, I. Foster, K. Gaither, A. Grimshaw, V. Hazlewood, S. Lathrop, D. Lifka, G. D. Peterson, R. Roskies, J. R. Scott, and N. Wilkins-Diehr, "XSEDE: Accelerating scientific discovery," Comput. Sci. Eng. 16(5), 62-74 (2014).

\section{Introduction}

Optical supercontinuum (SC) results from the synergy of several nonlinear and linear processes, all acting together [1-6]. Supercontinuum generation is these days finding applications in biomedical imaging, optical metrology, spectroscopy, and sensing, to mention a few $[1,4]$. In this regard, single-mode photonic crystal fibers with pre-engineered dispersion characteristics are currently commercially used in implementing spatially coherent ultrabright SC sources, spanning the wavelength range from ultraviolet to the near-infrared [2-5]. Given that most SC sources have so far relied almost exclusively on single mode or few mode fiber technologies, it will not be long before limits are reached in terms of output power capabilities, especially for applications where high spectral power densities are required. A possible avenue to overcome these hurdles could be through the use of large-area multimode fibers (MMFs). Multimode fibers, having been overlooked for decades, are nowadays making a strong comeback because of space-division multiplexing applications [7-9]. These fibers are by nature complex structures: they can support thousands of modes that can in turn interact among themselves in a variety of ways. Even though over the years, some of their 
nonlinear properties have been intermittently investigated [10-21], in general, the manner nonlinear processes unfold in such convoluted multimode environments still remains an issue that is largely unexplored.

Quite recently, the nonlinear "virtues" of nonlinear MMFs have been reconsidered in both the normal and anomalous dispersion regimes [22]. In this vein, the observation of multimode optical solitons in parabolic MMFs has been reported $[23,24]$ - thus confirming earlier predictions $[13,25]$. Supercontinuum generation has also been successfully demonstrated in graded index MMFs by launching ultra-short pulses in the anomalous dispersion region (1550 $\mathrm{nm})[26,27]$. In these experiments, discrete spectral components were observed in the visible domain - features that were subsequently explained through the interplay between spatiotemporal soliton oscillations and dispersive waves that are only possible in parabolic fibers [27]. In addition, efficient supercontinuum generation from the visible to near-infrared (when pumped in the normal dispersion regime, $1064 \mathrm{~nm}$ ) was reported in low DGD (differential group delay) parabolic MMFs [28] by making use of a newly observed mechanism - better known as geometric parametric instability [29,30]. Such MMF supercontinuum sources could potentially display spectral densities that are orders of magnitudes higher than those currently obtained in single-mode fiber systems [28]. Beam clean-up was also observed for the first time during SC generation [28,31]- an effect that is yet not fully understood. These latter studies all indicate that MMFs can provide a rich setting where nonlinear effects can be potentially molded by exploiting the sheer complexity offered by a multitude of wave-mixing paths. Of importance will be to assess to what extent one can shape the output of such experiments through new degrees of freedom.

In this paper, we show that the input beam modal composition can provide two additional degrees of freedom in tailoring the output spectral content resulting from nonlinear interactions in graded-index multimode fibers. These two parameters directly stem from the indices $(l, m)$ of the supported mode group $L P_{l m}$. Our study suggests that soliton fission and emission effects as well as dispersive wave generation, known to play an important role during SC, critically depend on the way the modes of this fiber are initially excited. Unlike single mode fibers, initial beam conditions tend to affect multimode soliton velocities and their energy content. Numerical simulations based on a gUPPE approach [32,33] are in good agreement with previously reported experimental observations, carried out at $1550 \mathrm{~nm}$ [26].

\section{Theory and method}

To demonstrate the effect initial spatial conditions, have on the generated SC spectra in heavily multimoded fibers, we first perform a series of numerical studies. The silica fiber under investigation is assumed to have a core diameter of $2 \mathrm{a}=62.5 \mu \mathrm{m}$ and a parabolic index profile (so as to minimize modal walk-off) with a maximum numerical aperture $N A=0.275$. This MMF is excited at $1550 \mathrm{~nm}$ where the dispersion is anomalous and at which wavelength this structure is expected to support $\sim 300$ modes. Given that the number of propagating modes scales as $1 / \lambda^{2}$, one would expect thousands of modes especially in the visible range. Clearly, simulating in full this complex environment on a mode-by-mode basis becomes quickly impractical, if not impossible. Each mode requires a pre-computed dispersion curve, and each nonlinear interaction is described by a pre-calculated tensor requiring billions or trillions of non-zero multi-dimensional integrals. Even if these could be computed, coupledmode models cannot accurately describe the full wavelength-dependence of modes and nonlinear interactions, especially near cut-off as occurs for many modes involved in our broadband spectra. In addition, a mode-by-mode methodology will fail to capture the ensued dynamics in such a broad parabolic index profile, even at moderate power levels where weak self-focusing effects are expected to present themselves. Hence, while such models can provide insight, for highly-multimode propagation especially, a full theoretical understanding absolutely requires rigorous full-field simulations based on the gUPPE [32,33]. This 
unidirectional pulse propagator (UPPE) allows one to model supercontinuum generation in highly multimode MMFs in a global manner, accounting for all possible nonlinear effects. In this respect, the evolution of the vectorial electric field is comprehensively described by

$$
\partial_{z} \vec{E}\left(k_{\perp}, \omega, z\right)=i k_{z} \vec{E}\left(k_{\perp}, \omega, z\right)+\frac{i \omega^{2}}{2 \varepsilon_{0} c^{2} k_{z}} \vec{P}\left(k_{\perp}, \omega, z\right)-\frac{\omega}{2 \varepsilon_{0} c^{2} k_{z}} \vec{J}\left(k_{\perp}, \omega, z\right) .
$$

where $k_{z}\left(k_{\perp}, \omega\right)=\sqrt{\omega^{2} \varepsilon(\omega) / c^{2}-k_{x}^{2}-k_{y}^{2}}$ and $\varepsilon(\omega)$ is the frequency dependent permittivity of the medium with $\mathrm{c}$ representing the speed of light. The waveguide is represented as a static contribution to polarization $\vec{P}$, and is implemented as an additional module for the simulator. Equation (1) implicitly considers diffraction/waveguiding processes as well as dispersion and mode walk-off effects. Moreover, all the nonlinear interactions are included in the polarization and current terms, represented by $\vec{P}\left(k_{\perp}, \omega, z\right)$ and $\vec{J}\left(k_{\perp}, \omega, z\right)$ in (1), respectively. For the task at hand, the UPPE core is primed to account for all nonlinear phenomena such as self-phase and cross-phase modulation, four-wave mixing, third harmonic generation, shock-effects, Raman, etc. The nonlinear Kerr coefficient is $n_{2}=2.9 \times 10^{-20} \mathrm{~m}^{2} / \mathrm{W}$ and its Raman fraction is taken here to be $\mathrm{f}=0.18$ in the standard two-parameter response model [34]. The wavelength dependence of the refractive index is provided through an appropriate Sellmeier series. In our studies, the current term (accounting for possible plasma and filamentation effects) in Eq. (1) played a negligible role.

\section{Numerical and experimental results}
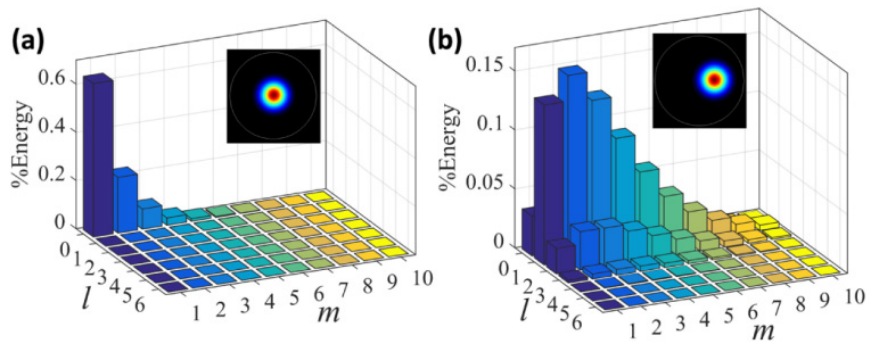

Fig. 1. (a) Energy distribution among the $L P_{l m}$ modes of a parabolic fiber when excited onaxis. In this case, only the $L P_{0 m}$ modes are populated with the fundamental mode taking most of the energy. (b) Modal population when the system is excited off-axis. For this input, considerable energy resides in the $L P_{l m}$ set.

In all our simulations, we assume that the input pulse energy is $E_{\text {in }} \approx 150 \mathrm{~nJ}$. The temporal pulse width is $\tau \approx 500 f_{s}$ (FWHM) and the minimum waist spot-size (where the intensity drops by $e^{-2}$ ) of the input circular Gaussian beam is $w=15 \mu \mathrm{m}$. Evidently, different modal groups $L P_{l m}$ will be excited depending on the position of the input Gaussian beam. In this case, the modal content can be directly obtained by projecting the input field profile on the orthogonal Gauss-Laguerre base functions. For example, if the beam launched is centered, only modes from the $L P_{0 m}$ group will be excited, i.e. having zero orbital angular momentum. To some extent, this modal population is akin to that associated with a vacuum squeezed or coherent state. For the parabolic fiber under consideration, the modal composition corresponding to an on-axis Gaussian beam excitation is shown in Fig. 1(a). Meanwhile, if this same Gaussian beam is shifted by $10 \mu \mathrm{m}$ with respect to the fiber center, the modal composition is significantly altered, as depicted in Fig. 1(b). In this latter scenario, most of the energy no longer resides in the fundamental mode. Instead, the mode content does not 
monotonically decrease with $m$ and in addition, modes with angular momenta are now involved (with $L P_{1 m}$ contributing the most).

Under the aforementioned initial conditions, the evolution of the SC spectrum, as obtained from gUPPE simulations is shown in Figs. 2. The supercontinuum features in Fig. 2(a) result from an on-axis excitation, (corresponding to Fig. 1(a)) while those in Fig. 2(b) from an offaxis input (Fig. 1(b)). Overall, these two figures show there is a significant difference in the way SC develops in this MMF, depending on the modal groups initially excited. If the fiber is excited on-axis with a spot-size different than that of the fundamental mode, the beam experiences periodic contractions and expansions. Meanwhile, if the Gaussian wavefront is launched off-axis, in addition to the aforementioned effects, the beam tends to oscillate around the fiber center [35].

As Fig. 2 indicates, in both cases, the spectrum rapidly broadens with propagation distance. Three stages of spectral evolution are clear in this figure. During the first stage, the spectrum gradually expands and the temporal and spatial profiles of the pulsed beam continuously contract. In addition, the periodic compression/expansion of light that results from modal interference, an innate property of parabolic-index optical fibers, leads to a geometric parametric instability (GPI) which manifests itself as narrow line sidebands symmetrically located around the pump frequency $\omega_{p}$ [28-30]. The angular frequency of the generated mth sideband can be obtained from $\omega_{S B_{m}} \simeq \omega_{p} \pm \sqrt{m} \omega_{G}$, where $\omega_{G}^{2}=2 \delta /\left|k_{0}^{\prime \prime}\right|$. Here $\delta=a^{-1} \sqrt{2 \Delta}$ represents the spacing between the propagation constant eigenvalues, $\left|k_{0}^{\prime \prime}\right|$ is the fiber dispersion at the pump wavelength, and $\Delta=\left(n_{\text {core }}-n_{\text {clad }}\right) / n_{\text {core }}$. In our experiments, $\Delta=0.0165$, and $\left|k_{0}^{\prime \prime}\right|=2.8 \times 10^{-26} \mathrm{~m}^{-1} \mathrm{~s}^{2}$ For these parameters, the first three visible sidebands are expected to appear at 296,338 , and $371 \mathrm{THz}$ while the first NIR sideband at $91.2 \mathrm{THz}$. These sidebands are easily discernable in Fig. 2(b). Interestingly, however, this is not the case in Fig. 2(a). This can be understood by keeping in mind that the GPI process is a direct byproduct of modal interference. In the first case, when the system is excited on-axis with a spot size close to that of a fundamental mode (most of the energy resides in $L P_{01}$ ), the ensued GPI is weak and hence little energy is transferred to the sidebands. On the other hand, when the MMF is excited off-axis, the compression/expansion cycles are more severe and the GPI gain is therefore higher, as indicated in Fig. 2(b).
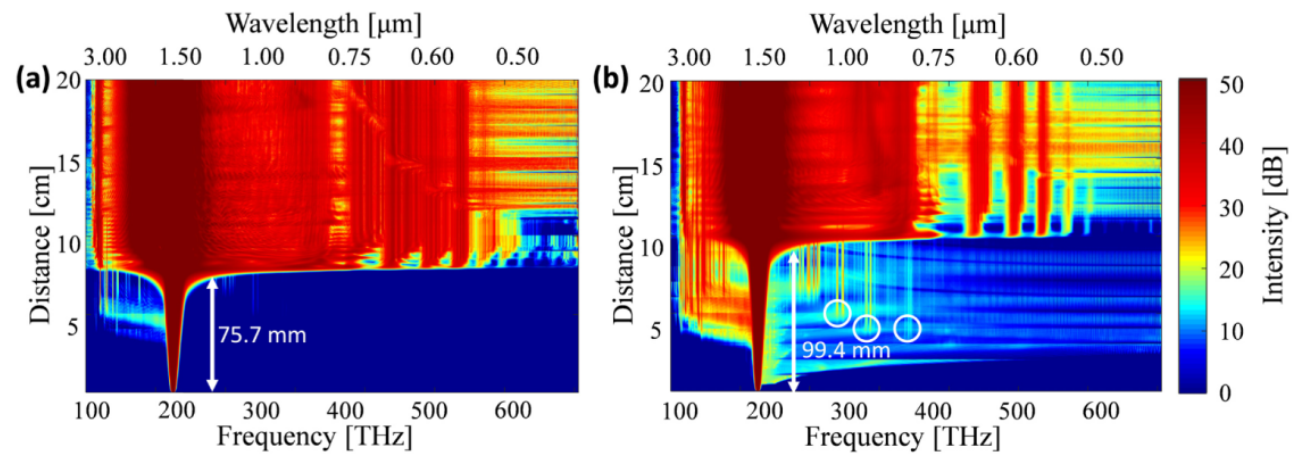

Fig. 2. (a) Evolution of the supercontinuum spectrum in a $20 \mathrm{~cm}$ long parabolic MMF when the pulse energy is $150 \mathrm{~nJ}$ for (a) an on-axis excitation (b) off-axis input (offset by $10 \mu \mathrm{m}$ ). In all cases three stages are apparent: (i) initial spectral broadening, (ii) soliton fission, and (iii) soliton and dispersive wave propagation. The respective distances where soliton fission occurs are also shown. In (b) the circles denote the onset of the first three GPI sidebands. The pump wavelength is $1550 \mathrm{~nm}$. 
During the second stage, the input pulse, having enough energy to support multiple solitons, breaks into a series of distinct fundamental multimode soliton components. Each soliton, generated in this soliton fission stage (in the anomalous dispersion regime) has different properties or modal composition. Each produces linear dispersive waves in the normal dispersive region with correspondingly different characteristics. The distance at which this process occurs, not only depends on the input energy and dispersion, but also on the initial spatial conditions. In fact, since each spatial mode satisfies a different dispersion relation, the way the input energy is distributed among modes determines the effective dispersion and thus directly affects the fission distance. As it can be seen in Fig. 2(a), for onaxis excitation, the pulse undergoes fission after $76 \mathrm{~mm}$ of propagation, while for the off-axis case it takes place at $99 \mathrm{~mm}$ (Fig. 2(b)).

During the third stage, the co-propagation of solitons and dispersive waves in the MMF is responsible for supercontinuum generation, through the interplay of all the aforementioned processes. The spectra developed after $20 \mathrm{~cm}$ of propagation, corresponding to the on-axis and off-axis initial conditions of Fig. 1, are depicted in Fig. 3. Both Figs. 2 and 3 reveal significant differences in the resulting spectral features, suggesting that initial conditions indeed matter in supercontinuum generation. Launching the pulse on-axis leads to more intense multimode solitons, capable of experiencing stronger Raman self-frequency shifts in the infrared region. The gradual redshifting of solitons is accompanied by a gradual drift of their dispersive waves toward shorter wavelengths. This redshifting effect along with the ensuing FWM mechanism results into a rather flat spectrum. On the other hand, exciting the fiber off-axis produces distinct dispersive wave components in the visible (Fig. 3, red curve) that do not drift toward higher frequencies since the generated solitons are less susceptible to redshifting.

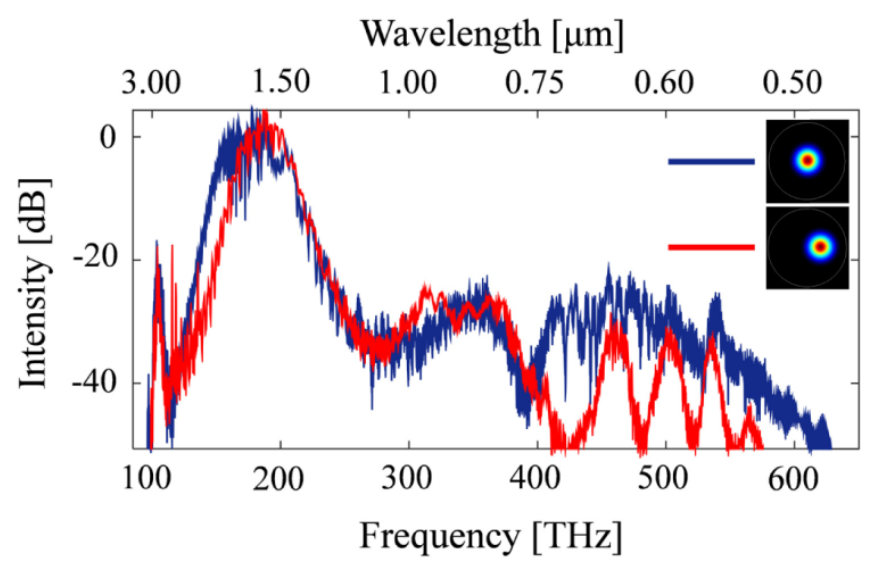

Fig. 3. Comparison of the supercontinuum spectra features produced in an anomalously dispersive parabolic MMF for on-axis (blue) versus off-axis (red) excitation conditions. The propagation distance is $20 \mathrm{~cm}$ and the pulse width is $500 \mathrm{fs}$. All other parameters are the same as Fig. 2.

This sensitivity of the emergent spectral features to the initial modal composition or spatial launching conditions can only be understood by taking a closer look at the temporal and spatial behavior of the elements involved in this process (Figs. 4(a)-4(f)). Figures 4(b) and (d) show the temporal evolution of the input pulse (500 fs, $150 \mathrm{~nJ}$ ) in our parabolic MMF during the first $20 \mathrm{~cm}$ of propagation distance, under on-axis and off-axis excitation conditions, respectively. As can be inferred from the slopes of the traces, which are representatives of wavepacket velocities with respect to the moving frame, the fission generated solitons from an on-axis input propagate much slower than those resulting from an off-axis excitation. For these two scenarios, the spatial intensity distribution associated with 
the slowest and more pronounced solitons is depicted by the insets in Figs. 4(e) and 4(f). For clarity, these same figures also provide $x$-cross-sections of these intensity profiles (red curves). Moreover, the modal composition of these primary solitons has been numerically estimated by projecting their spatial fields on the constituent $L P_{l m}$ modes of the MMF at that particular wavelength (energy distributions in Figs. 4(e) and 4(f)). These results demonstrate that, the solitons generated from on-axis excitation reside mostly in the fundamental mode while higher order modes like $L P_{02}, L P_{11}$, etc. jointly share only 10 percent of its energy. However, for off-axis excitation conditions, the resulting solitons are heavily multimoded, with more than 40 percent of their energy distributed among higher-order spatial modes. Since the fundamental mode in the former case, dominates in terms of energy, the Raman self-frequency shift is expected to be more pronounced. As indicated in previous studies [17], this is because the $L P_{01}$ mode typically enjoys a higher Raman gain - a direct by-product of the overlap integrals. This explains why the generated solitons under off-axis conditions experience less Raman deceleration and hence move faster. In addition, the modes involved, as initially dictated by launching conditions, dynamically exchange energy during propagation as a result of wave-mixing and Raman processes. Figures 4(a) and 4(c) show the temporal features at the output of the fiber. In both cases, the solitons have a pulse-width of 18-20 fs. However, the energy each soliton carries depends again on initial conditions. In fact, for an on-axis input, the first generated soliton contains almost $40 \%$ of the total energy.
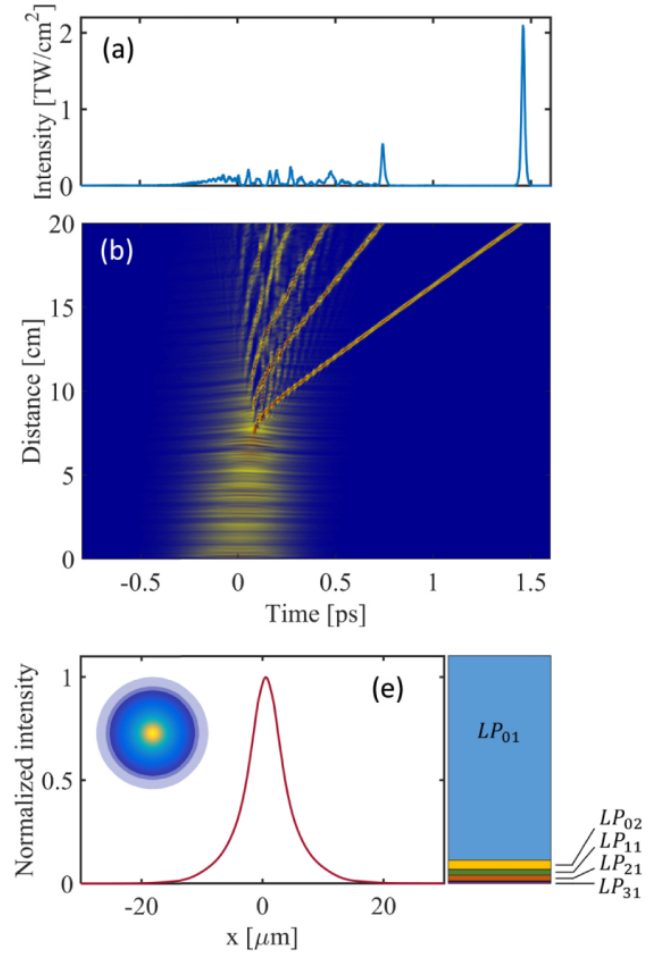
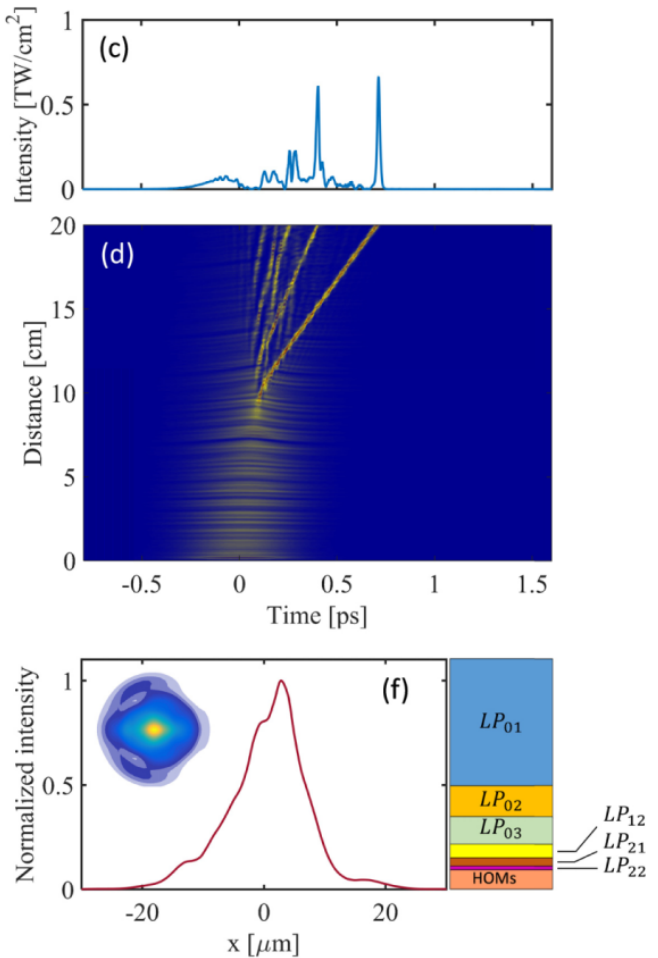

Fig. 4. (a) Resulting temporal features after $20 \mathrm{~cm}$ in a parabolic MMF when excited on axis. (b) Temporal evolution of the initial $500 \mathrm{fs}$ pulse $(150 \mathrm{~nJ})$ corresponding to (a). The emergence of slow solitons is apparent. (c) and (d) Same as in (a) and (b), respectively, for off-axis excitation. (e) The spatial intensity profile and its $x$-cross section corresponding to the slowest dominant soliton at the end of the fiber, when illuminated with an on-axis Gaussian beam. (f) Same as in (e) for off-axis launching conditions, where the soliton-beam experiences transverse oscillations during propagation. 
The simulations show good agreement with experiments [26]. In our experiments, an amplified fiber laser operating at $1550 \mathrm{~nm}$ and emitting pulses with a $\sim 500 \mathrm{fs}$ pulse-width, was coupled into $\sim 1 \mathrm{~m}$ long fibers with the same parameters used in our numerical studies. By translating the fiber with respect to the optical lens, the initial conditions were varied. The Gaussian mode field diameter at the input was about $30 \mu \mathrm{m}$. Figures 5(a) and 5(b) show, respectively, the NIR and visible portions of the output spectra resulting from an on-axis excitation. Figures 5(c) and 5(d), on the other hand, depict the spectra for the off-axis case ( $10-15 \mu \mathrm{m}$ displacement). The near-field beam profile, integrated over all wavelengths, corresponding to each of these measurements is shown on the right of each figure. In agreement with our numerical results, we observe that most of the energy resides in the fundamental mode, thus causing significant redshifting towards NIR from the generated solitons (Fig. 5(a)). Meanwhile, by moving the input beam away from the center, the frequency generation in the NIR region is considerably subdued (Fig. 5(c)). As also predicted by our simulations, the visible component of the spectrum, depicted in Fig. 5(b) for on-axis, exhibits appreciable frequency generation over a broad range while the output spectrum resulting from an off-axis excitation displays instead a series of distinct peaks (Fig. 5(d)).

(a)
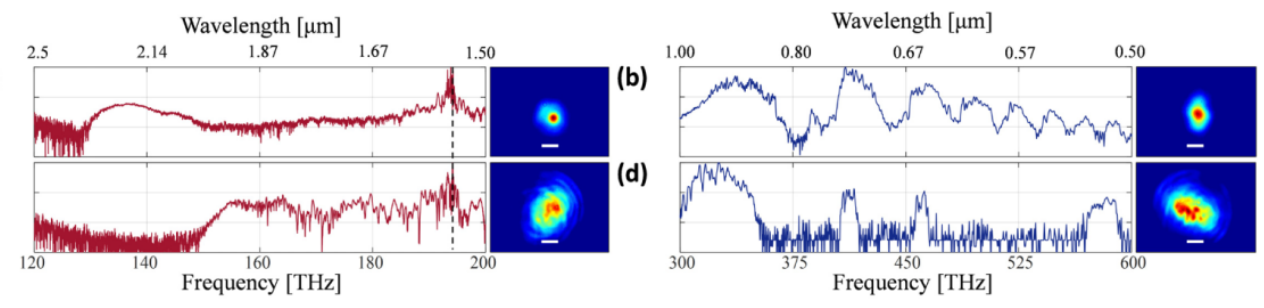

Fig. 5. Experimentally measured (a) NIR and (b) visible supercontinuum spectrum for on-axis excitation together with generated transverse output intensity profiles after $1 \mathrm{~m}$ of propagation. (c) and (d), Same as in (a) and (b) for an off-axis excitation. In all cases, each division represents a $10 \mathrm{~dB}$ variation. The scale bars in the insets represent $20 \mu \mathrm{m}$.

Our results clearly indicate that changing the initial spatial conditions can have a significant effect on the spatial and temporal evolution of the multimoded field which accordingly alters the frequency generation process. Thus far, we have mostly studied the effect of $L P_{0 m}$ (with the fundamental mode dominating) and $L P_{1 m}$ modes on the output spectrum. Yet, a plethora of other initial conditions can in principle be synthesized, with each one leaving a different imprint in the output. As an example, we here theoretically investigate two other possibilities. In the first case, the fiber is illuminated with a ring pattern having a radius of $25 \mu \mathrm{m}$ and a FWHM of $4.5 \mu \mathrm{m}$. Under these conditions, $80 \%$ of the total energy is distributed among the $L P_{06}, L P_{07}$, and $L P_{08}$ group of modes, with the $L P_{07}$ possessing most of the energy. The share of the first five $L P_{0 m}$ modes, including the fundamental, remains below $5 \%$. The peak intensity and energy of the pulses used are the same as in Figs. 2-5. Figures $6(\mathrm{a})$ and $6(\mathrm{~b})$ show the NIR and visible portions of the output spectrum after $20 \mathrm{~cm}$ of propagation. As it can be inferred from Fig. 6(a), the self-frequency shift of the generated multimoded solitons is rather small. This can be attributed to the fact that higher $L P_{0 m}$ modes tend to experience lower Raman gains. Interestingly, however, this same modal composition leads to a considerable enhancement of supercontinuum in the visible portion of the spectrum (400-600 THz), as shown in Fig. 6(b). This enhancement is closely related to a more efficient generation of dispersive waves via Raman solitons. Meanwhile, the component between 300 and $400 \mathrm{THz}$ - arising from dispersive waves emitted at the pump wavelength (PDWs) - is suppressed. 


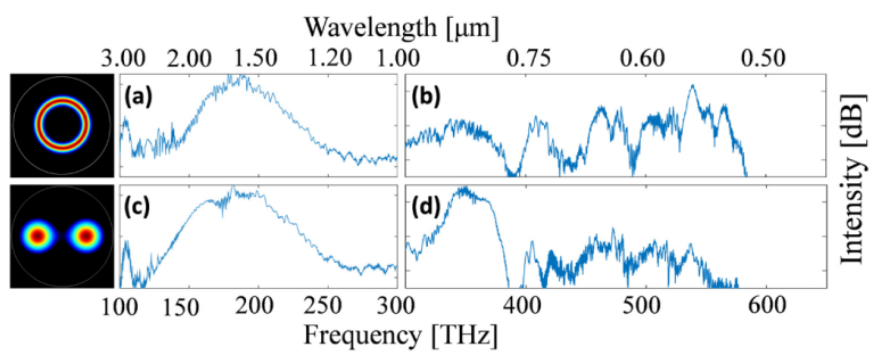

Fig. 6. Generated (a) NIR and (b) visible portion of the spectrum when the fiber is excited by a ring beam. Same as in (c) and (d) when using a two-spot excitation. Each division in (a) and (c) represents a $20 \mathrm{~dB}$ differential while in (b) and (d) a $10 \mathrm{~dB}$ variation.

As a second example, we consider the case of symmetrically exciting the fiber with two spots in phase, akin to a quantum cat-state $|\psi+|-\psi$. This state results into the elimination of all $L P_{2 k-1, m}$ modes where $k$ is a positive integer. In our simulations, the fiber is excited with two Gaussian beams, separated by $20 \mu \mathrm{m}$. Each of these two displaced Gaussian beams is identical in size with those used in Figs. 2-4 and carries half of the total energy $(75 \mathrm{~nJ})$. In this arrangement, the energy is distributed among the $L P_{0 m}$ and $L P_{2 m}$ group of modes, with the fundamental mode having the largest share. Figure 6(c) shows the resulting NIR supercontinuum spectrum. As expected, the Raman process is now more pronounced given that a larger portion of the energy is injected in the fundamental mode. On the other hand, the visible section of the spectrum, shown in Fig. 6(d), indicates that this symmetric excitation leads to a notable enhancement in the $300-400 \mathrm{THz}$ band (arising from PDWs) while the higher frequencies are subdued.

Figure 7 shows experimental results corresponding to four different initial spatial conditions. In all cases, the generated supercontinuum spectrum is highly sensitive to the modal groups initially excited. The integrated output beam profiles in the visible and NIR are also depicted. While in Fig. 7(b) the spectral features are comb-like, in (d) they are more broad and flat, similar to observations in our simulations (Figs. 6(b) and 6(d)). Similar conclusions also hold for the experimental observations of Figs. 7(a) and 7(c).
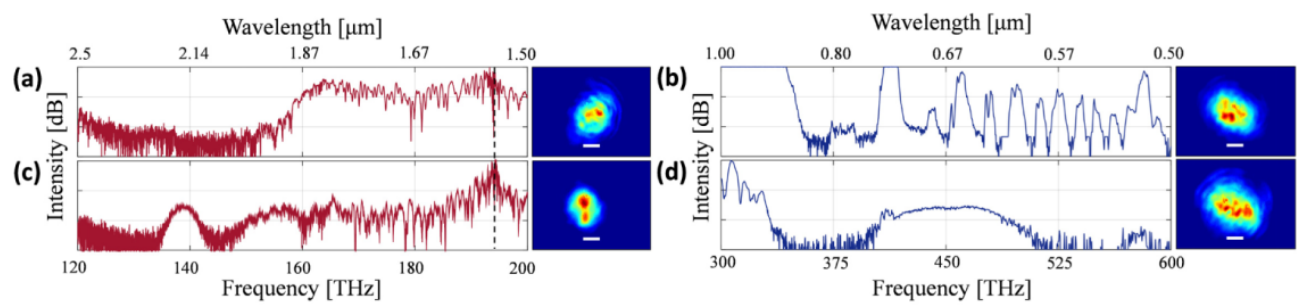

Fig. 7. Experimentally measured NIR and visible supercontinuum spectra for four different initial spatial conditions together with generated transverse output intensity profiles after $1 \mathrm{~m}$ of propagation. In all cases the pulse energy is $150 \mathrm{~nJ}$. In all cases, each division represents a 10 $\mathrm{dB}$ variation. The scale bars in the insets represent $20 \mu \mathrm{m}$.

\section{Conclusion}

In conclusion, we have shown that parabolic multimode fibers can provide a versatile platform for tailoring supercontinuum generation. Experiments and simulations carried out in the anomalous dispersive region indicate that the modal composition (energy distribution and phase relationship among eigenmodes) of the input beam plays an important role in altering the output spectrum. This is accomplished through the interplay of soliton fission procresses, Raman, dispersive wave generation and four-wave mixing. Our results could pave the way for 


\section{Optics EXPRESS}

new classes of optical sources with pre-engineered spectra and unprecedented high spectral densities.

\section{Funding}

This work was supported by the Office of Naval Research (ONR) (MURI N00014-13-10649), HEL-JTO (W911NF-12-1-0450), Army Research Office (ARO) (W911NF-12-1-0450) and the Air Force Office of Scientific Research (AFOSR) FA9550-15-10041.This work was also partially funded by the Qatar National Research Fund (grant: NPRP 9-020-1-006)

\section{Acknowledgment}

This work acknowledges the support of the Extreme Science and Engineering Discovery Environment (XSEDE) [36], which is supported by National Science Foundation grant number ACI-1053575. The University of Central Florida Stokes Advanced Research Computing Center (UCF ARCC) was also partially used to perform simulations. LGW acknowledges partial support from the National Sciences and Engineering Research Council of Canada. 